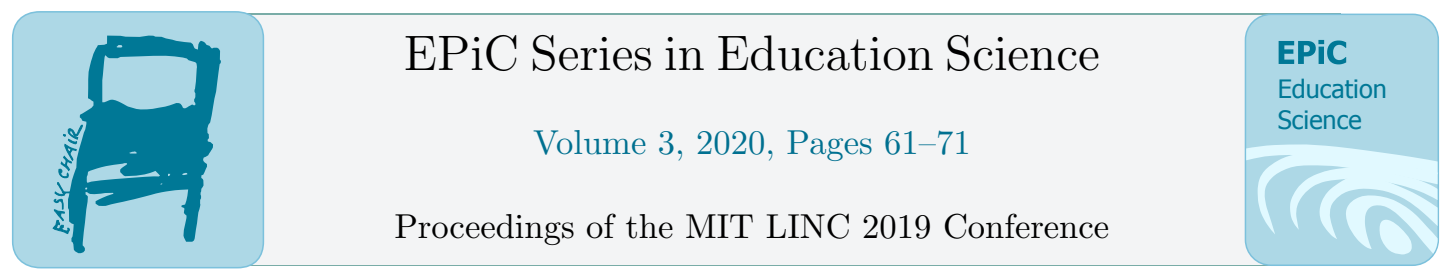

\title{
The Value of the We Love Reading Program for Executive Functions In Jordanian Children
}

\author{
Dr. Rana Dajani ${ }^{1}$, Alya Al Sager ${ }^{2}$, Diego Placido $^{3}$, Dr. Dima Amso ${ }^{4}$ \\ ${ }^{1}$ Hashemite University \\ rdajani@hu.edu.jo \\ ${ }^{2}$ Brown University \\ alya_al_sager@brown.edu \\ ${ }^{3}$ Brown Univeristy \\ diego_placido@brown.edu \\ ${ }^{4}$ Brown Univeristy \\ dima_amso@brown.edu
}

\begin{abstract}
Early childhood enrichment opportunities have been shown to shape Executive Functions (EFs), which in turn play a critical role in the development of academic skills, including school readiness and future educational achievement and mobility. We partnered with We Love Reading, a Jordan-based organization designed to promote reading for pleasure among children, in order to examine the impact of the WLR read-aloud method on executive functions in children. Children completed a battery of executive functions tasks and parents filled out behavioral and demographic assessments of their children. Over a six month interval with the WLR program, we found that the number of books in the home and the number of children that considered reading as a hobby had increased. Changes in reading in the home from baseline to post-WLR also predicted larger improvements in executive functions, and particularly for younger children and for families who reported lower family income.
\end{abstract}

C. Urrea (ed.), LINC 2019 (EPiC Series in Education Science, vol. 3), pp. 61-71 


\section{Introduction}

Executive Functions (EFs) are a group of related developing processes that are relevant to planning, decision-making, and regulating one's behavior and emotions [1]. Working memory is an executive function that involves holding multiple pieces of information in mind and acting in accord with high-level goals. Inhibitory control involves suppressing inappropriate thoughts or actions. Flexible task switching refers to maintaining multiple rules in mind and responding flexibly when rules change. These skills undergo important development during early childhood [2, 3] and are shaped by enriching experiences in a child's home and school environment [4]. Critically, EFs shape children's progress in school, and have been shown to mediate the relationship between socioeconomic status (SES), a proxy for enriching experiences, and academic achievement [5]. Thus, programming that improves EFs in early childhood provides great benefit for long-term outcomes, including school readiness and future educational success. Here, we examined the impact of reading for pleasure, an informal educational practice, on executive functions in 4-8 year-old Jordanian children.

Both the availability of books and the process of reading to children have received a great deal of research attention, in that many have argued that exposure to language, or even multiple languages, is critical for EF development [6]. However, recent work has shed new light on the mechanisms that drive the 30-million-word gap between children of higher and lower SES backgrounds. Romeo et al. (2018) found that these effects are largely driven by the number of conversational turns between the caregiver and child, rather than the number of adult words spoken to the child [7]. That is, engaging with the child in the context of reading, rather than the reading process itself, may be a driving force for positive change in the processes that underlie learning and achievement.

Most Arab countries lack a reading culture [8]. For instance, the Arab Thought Foundation Fikr found that Arab children read for an average of 6 minutes per year compared to their Western counterparts, at 12,000 minutes per year [8]. We Love Reading (WLR) is run by an independent non-profit organization called Taghyeer. WLR is designed to engage children in reading for pleasure and involves training local ambassadors to hold routine read-aloud sessions for children ages 0-10 years old in public spaces of their neighborhoods. WLR then provides children with the opportunity to take the books home to read, making use of what is called a 'living library.' WLR is quickly becoming an established social movement, and currently 
operates in 46 countries, with over 4,000 reading ambassadors reaching 440,000 children. The program is simple, sustainable, local, cost efficient, culturally-sensitive, and child-focused. WLR engages the community and parents with the child, offering various sources of motivation and social scaffolding known to be powerful in early childhood resilience [9] and particularly so in collectivist cultures [10]. WLR chooses books that are age-appropriate, attractive, neutral in content, and in the native language of the child.

A stated goal of program is to empower the child to be an agent of change in their home environment. The claim is that excitement at public readings would result in changes in reading patterns at home with parents. We trained local school teachers to read to children using the WLR read-aloud method. Before and after six months of WLR public readings, we examined (1) motivation around books and reading in the home and (2) whether change in home reading patterns had an impact on EFs.

\section{Methods}

\subsection{Participants}

The final sample of participants in this study included a total of $N=606-8$ year-old Jordanian children ( $M$ age $=7.06$ years old, $S D=0.70 ; 22$ females, 38 males) that participated in two sessions spaced approximately 6 months apart $(M=5.5$ months, $S D=.16$ ). An additional 7 children were tested but not included in the final data analysis because their baseline and post-WLR testing sessions were spaced 3 SDs below (example 4.68 months) or above (example 10.56 months) the remainder of the sample. The Brown University Institutional Review Board approved the study procedure and parental consent was obtained prior to testing. Families were compensated (\$10) for their participation. Exclusionary criteria from participating in the study included severe birth complications, other known developmental disorders, history of neurological problems or head injury, gross malformations of the skull, or for known uncorrected visual or auditory impairments (e.g., vision or hearing loss, amblyopia). Table 1 shows basic participant testing, participation, and demographic information. 
Table 1

Descriptive Demographic Statistics of Total Sample at Baseline

\begin{tabular}{lccc}
\hline & Mean & Standard Deviation & Range (min, max) \\
\hline Gender (frequency) & 38 & & \\
Male & 22 & & \\
Female & 7.06 & & \\
Age (years) & 1.83 & 0.70 & $(5.9,8.6)$ \\
Grade in School & 5.53 & .642 & $(1,3)$ \\
Time Between Assessments (months) & 16.85 & .16 & $(5.2,6)$ \\
WLR Sessions Attended & 14.47 & 1.40 & $(14,19)$ \\
Maternal Education (years) & 14.00 & 2.34 & $(8,20)$ \\
Paternal Education (years) & 6,011 & 2.85 & $(1,20)$ \\
Family Income (JD) & & 5386 & $(300,17,000)$ \\
\hline
\end{tabular}

\subsection{Design and Procedure}

WLR sessions took place in two school locations in Jordan. The rationale and explanation of the program was provided in information leaflets in Arabic and distributed to the parents. Participants completed the baseline assessment before the WLR public reading sessions and repeated the same set of assessments approximately 6 months post program initiation. Both parents and children were present for the two testing sessions. While parents filled out the demographic forms, children completed the computer EF tasks. Within-group variability in change of EFs was examined as a function of questions that probed the number of children's books in the home and motivation around reading with parents before and after WLR. Any changes in reading practices and attitudes in the home environment were spontaneous and not controlled or mandated by the researchers. These data are taken from a broad set of questionnaires and tasks included in the battery.

\subsection{EF Laptop Task Procedure}

The tasks and games were presented on a PC laptop to each participant. Children were seated in a quiet space with the laptop on a desk or table in front of them. A cover placed on the keyboard with only the D and K keys cut out. Children were asked to rest their pointer fingers on those keys during the games. In the Working Memory (WM) Task, children saw a fixation cross, followed by a heart icon either to the right or to the left of the fixation. They were instructed to hold one single rule in mind, "Press the button on the same side as the heart when it appears." On the Inhibitory Control Task, children saw a flower icon appearing to the right or left of the fixation cross. They were taught to press the button on the opposite side of the flower icon. That is, they had to both hold the new rule in mind and inhibit a prepotent response to the visual presentation to respond correctly. On the Mixed/Switching Task, the two working memory rules were mixed. Children saw both the hearts and flowers trials. Thus, they had to hold two rules in mind and switch between them. Task order 
was fixed at Working Memory Task, Inhibitory Control Task, and Mixed/Switching Tasks. The Working Memory and Inhibitory Control Tasks had 12 trials each, and the final 33 trials were a mix of hearts and flowers in the Mixed/Task Switching. Within each task, stimuli were presented for $1500 \mathrm{~ms}$ with an inter-stimulus interval of $1000 \mathrm{~ms}$. Trial order was counterbalanced. Children were given no feedback on performance after learning the rules.

\subsection{Behavioral and Demographic Assessments}
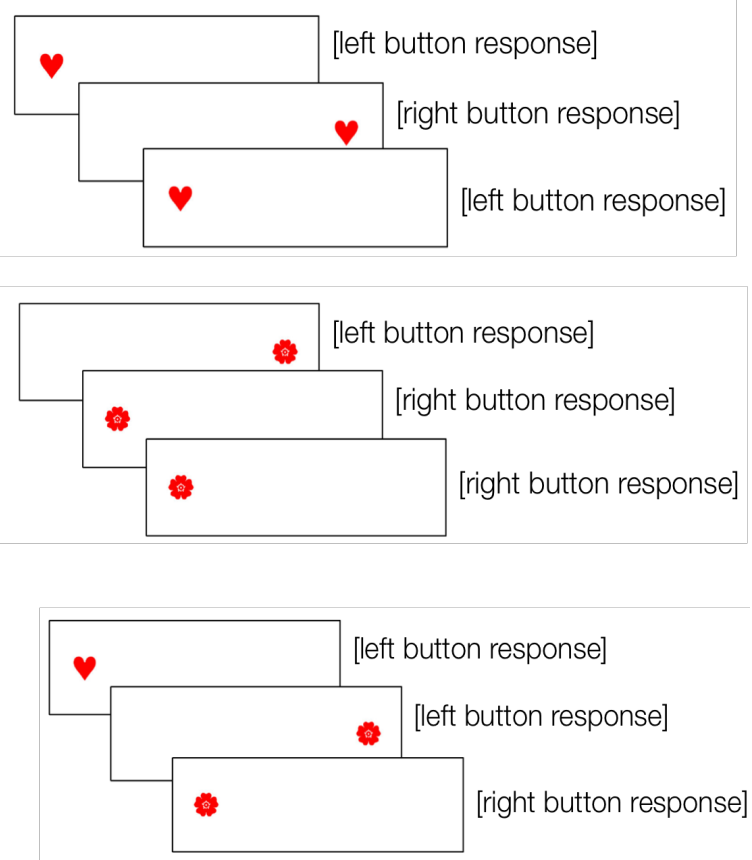

Figure 1: Illustrates, from top to bottom, Working Memory, Inhibitory Control and

Psychometric assessment tools, validated in Arabic, were completed at the schools with guidance from teachers when needed. Parents completed questions about their education level, occupation, and family income. Parents also completed the Home Observation for Measurement of the Environment-Short Form (HOME-SF) [11], which assess quality of the home environment, and the Child Behavior Checklist (CBCL) [12], which is a validated measure for children's emotional, behavioral, and social competence.

We also asked parents to fill out a Reading Diary for three months, where parents indicated their child's daily reading on a calendar. A total of 27 parents $(45 \%$ of the sample) reported no reading for the duration of the assessment on the Reading Diary. The remaining 33 parents $(55 \%)$ had a wide range of reported reading patterns. We asked if children whose parents reported reading at home on the Reading Diary differed from those who did not in any baseline characteristics that may impact the range of possible change from baseline in EFs. A logistic regression examining differences in the reading and no-reading families by Age at baseline, Executive Functions at baseline, Gender, Family Income at baseline, WLR Sessions attended at school, Internalizing Behaviors at baseline, Externalizing Behaviors at baseline, Paternal Education, and Maternal Education. Both Family Income, B = .182, Wald $\chi 2$ $=5.91, p<.05, \mathrm{OR}=1.2[1.04,1.39]$, and Internalizing Behaviors at baseline, $\mathrm{B}=-$ 
.03 , Wald $\chi 2=3.64, p=.057, \mathrm{OR}=.97,[.94,1.0]$ differed at baseline in families who reported reading at home in the Reading Diary. Internalizing behaviors are expressions of anxious, withdrawn, and depressed behaviors according to parent report on the CBCL. Children whose parents reported no reading on the Reading Diary have children with a lower internalizing score on the CBCL $(M=63.4, S D=26.77)$ and higher family income $(M=7760 \mathrm{JD}, S D=5580)$. Those who read to their children at home had lower family income $(M=4580 \mathrm{JD}, S D=4847)$ and children who scored higher on the CBCL metric of internalizing behaviors $(M=75.28, S D=21.70)$. These two otherwise uncorrelated variables, $r(57)=-.10, p=.94$, reflect differences both in the demographic of the sample and key characteristics of children at baseline that may impact EF change either independently or by interaction with WLR. They are thus included in our subsequent models of the impact of reading on change in EF performance.

\section{Results}

Executive Functions. We first asked whether EF performance changed over the course of the six-month interval. An EF Task (Working Memory, Inhibitory Control, Mixed/Switching) by Testing Time (Baseline, six months Post-WLR) resulted in an EF Task, $F(2,114)=150.16, p=.000$, Testing Time, $F(1,57)=17.09, p=.000$, but not a Task by Time interaction, $F(2,114)=.20, p=.82$. Figure 2 shows that children performed best on the single-rule Working Memory, followed by the Inhibitory Control, and finally the more difficult Mixed/Switching task. Indeed, performance in the Mixed/Switching task was at chance $(50 \%)$ at Baseline, $t(59)=-.284, p=.777$, and above chance 6 months later, $t(57)=3.53, p=.001$. Overall, performance on all three EF tasks improved over the six-month interval. 

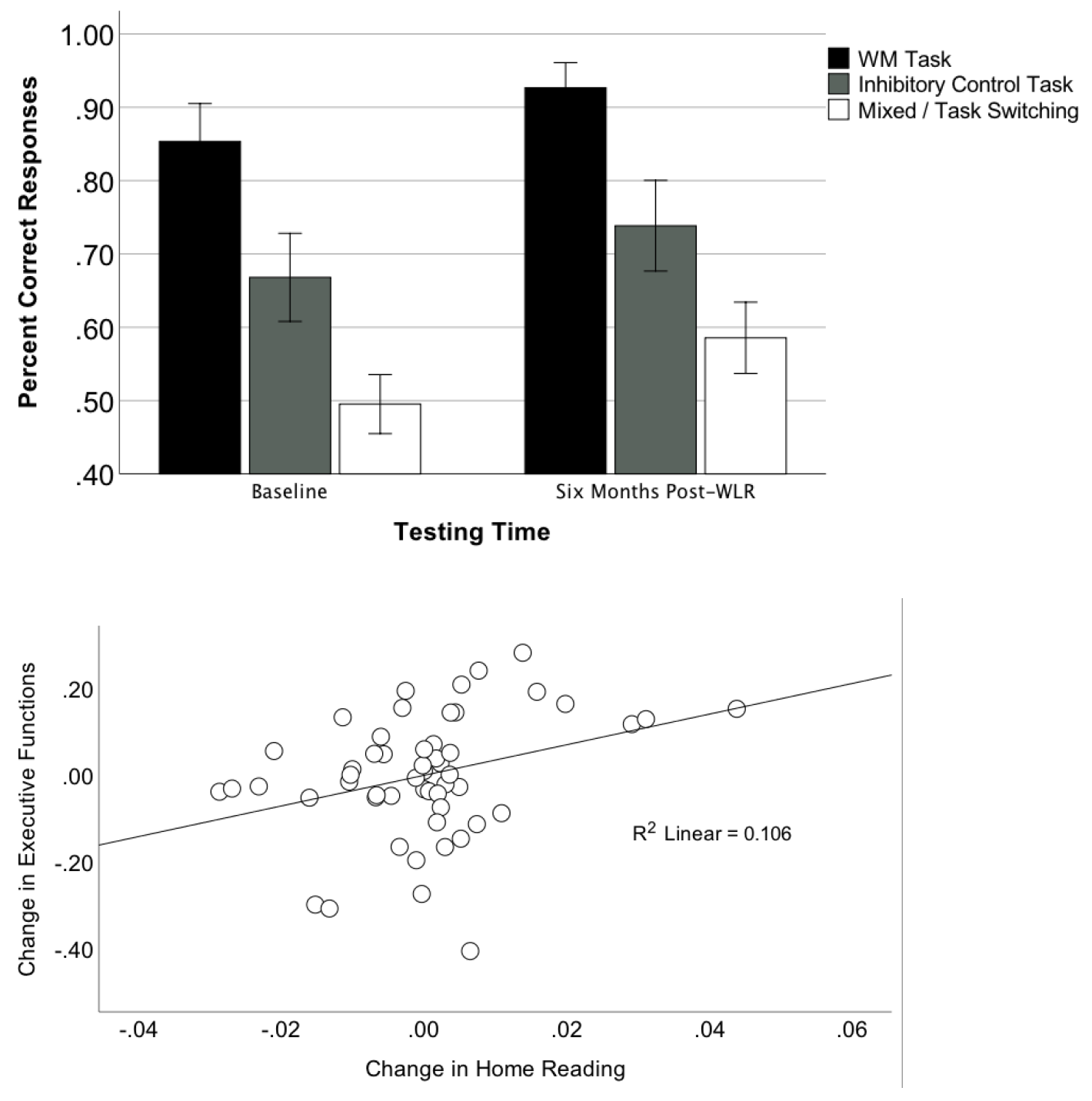

Figure 2. The top panel illustrates EF Task improvement in accuracy from Baseline to Post-WLR. The bottom panel is a partial probability plot showing that change in reading at home from Baseline to Post-WLR impacts this EF improvement.

Executive Functions and Reading. We first generated a metric of change in attitudes about reading at home. Parents filled out questionnaires at the baseline assessment and 
after 6 months of WLR public readings in schools. We asked parents four questions about children's reading attitudes and family practices in relation to reading using the HOME-SF and CBCL. The HOME-SF asked parents to estimate (1) how many books the child owns ( $1=$ None, $2=1$ to $9,3=10$ to 19 , and $4=20$ or more); (2) how often the parent reads aloud to the child $(1=$ Every Day, $2=$ Several times per week, $3=$ Several times per month, $4=$ Several times per year, 5 = Never/Don't know); and (3) how often the child reads for enjoyment (1= Every Day, $2=$ Several times per week, 3 = Several times per month, $4=$ Several times per year, $5=$ Never/Don't know). We subtracted values from baseline to post-WLR for each measure. We ran a factor analysis over the three HOME-SF measures and the data from the Reading Diary. This resulted in a single extracted component called Reading Change. This Reading Change measure is used to index change in reading at home from baseline and throughout the WLR 6 month interval. The CBCL included a question about whether the child considers reading a hobby (yes, no). The number of parents reporting that their children counted reading as a hobby changed from $13 \%$ of the sample to $23 \%$ of the sample after the WLR experience, $Z=-1.98, p<.05$. As well, parents reported significant increases in the number of books in the home from the baseline to the six months post-WLR assessment, $Z=-2.12 p=<.05$.

Our final and pertinent question was whether the change in home reading practices explained any of the change in EF processes in the six month WLR interval. Because there was a main effect of Testing Time, indicating that all EF tasks were showing a similar level of improvement over time, we averaged across all three EFs and generated an EF Change score (from baseline to post-WLR). We ran a linear regression with this value as the dependent variable. All predictors are listed in Table 3 and include the Reading Change score, Gender, Age at baseline, Family Income at baseline, Internalizing Behaviors at baseline, and the interactions among Reading Change and Age, Family Income, and Internalizing Behaviors at baseline. There was a significant effect of Home Reading Change Scores on improvements in EFs (Figure 2, bottom panel), and an interaction between this variable and Family Income, indicating a larger impact of Reading Change Scores on EFs in lower income children. Moreover, this effect further interacted with Age. Larger Reading Change scores had the largest positive impact for younger children at the lower end of the income range. 
Table 2 Summary of linear regression for variables predicting change in EFs

\begin{tabular}{lccc}
\hline & $B$ & SE $B$ & $\beta$ \\
\hline (Constant) & -.71 & .39 & .23 \\
Gender & .08 & .05 & .29 \\
Age In Years & .07 & 0.05 & $20.6^{*}$ \\
Reading Change & 3.5 & 1.60 & .25 \\
Internalizing Behavior & .002 & .001 & 1.41 \\
Family Income (JD) & .05 & .05 & -1.11 \\
Family Income x Age & .005 & .007 & $-19.78+$ \\
Reading Change x Age & -.48 & .24 & $-8.28^{*}$ \\
Family Income x Reading Change & -.21 & .08 & -16.53 \\
Family Income x Reading Change x & .03 & .01 & 15.68 \\
Age & & & .35 \\
Reading Change x Internalizing & -.04 & .02 & \\
Reading Change x Age x & .005 & .003 & \\
Internalizing & & & \\
Family Income x Reading Change x & .000 & .000 & \\
Internalizing x Age & & & \\
& & &
\end{tabular}

\section{Discussion and Conclusions}

We examined the impact of a reading for pleasure program, called We Love Reading, on change in EFs of 4-8 year-old children. The WLR read-aloud sessions seemed to influence spontaneous change in the number of books in the home and the number of children in the sample that consider reading a hobby. EFs showed expected developmental change in 4-8 year-old children in Jordan. Importantly, the change in reading attitudes and practices was related to improvement, in just six months, in executive functions development. This effect was particularly large for children from lower income homes. These data suggest that WLR read-aloud sessions impacted engagement with reading in the child's home environment. Reading is a form of enrichment that has multiple values for cognitive development. It involves interaction with parents at a time when parents are the primary source of rule-guided information for the child. It is an enrichment opportunity that allows turn-taking, verbal interaction with caregivers, practice with object forms (the written word), opportunities for imaginative play, creative thought, and learning others' perspective. All of these components of reading are important for supporting EFs development. 
EFs are a set of processes that govern context-appropriate thoughts and behaviors. They show a great deal of developmental change from three to about ten years of age and then again in adolescence [3]. EFs seem particularly relevant for early childhood education and success, and enrichment programs that can support their development are highly sought-after and informative [13]. Here we suggest a simple, sustainable and inexpensive opportunity to support developing EFs through reading for pleasure. The ultimate goal is to support life-long learning and academic achievement through natural and culturally-sensitive means. What is unique about WLR is that it is a local program that considers the significance of culture and context [14]. WLR offers an innovative simple solution that has the capacity to grow globally and sustainably. Reading is one medium, however, the cause is to encourage young children to realize that they have the potential and ability to think for themselves [15].

\section{References}

[1] Diamond, A. (2013). Executive Functions. Annual Review of Psychology, 64(1), 135-168. doi:10.1146/annurev-psych-113011-143750

[2] Diamond, A., Kirkham, N., \& Amso, D. (2002). Conditions under which young children can hold two rules in mind and inhibit a prepotent response. Developmental Psychology, 38(3), 352-362. doi:10.1037/0012-1649.38.3.352

[3] Davidson, M. C., Amso, D., Anderson, L. C., \& Diamond, A. (2006). Development of cognitive control and executive functions from 4 to 13 years: Evidence from manipulations of memory, inhibition, and task switching. Neuropsychologia, 44(11), 2037-2078. doi:https://doi.org/10.1016/j.neuropsychologia.2006.02.006

[4] Amso, D., Salhi, C., \& Badre, D. The relationship between cognitive enrichment and cognitive control: A systematic investigation of environmental influences on development through socioeconomic status. Developmental psychobiology, O(0). doi:doi:10.1002/dev.21794 [5] Lawson, G. M., \& Farah, M. J. (2017). Executive function as a mediator between SES and

academic achievement throughout childhood. International Journal of Behavioral

Development, 41(1), 94-104. doi:10.1177/0165025415603489

[6] Brito, N. H., \& Noble, K. G. (2014). Socioeconomic status and structural brain development.

Frontiers in Neuroscience, 8(276). doi:10.3389/fnins.2014.00276

[7] Romeo, R. R., Leonard, J. A., Robinson, S. T., West, M. R., Mackey, A. P., Rowe, M. L., \&

Gabrieli, J. D. E. (2018). Beyond the 30-Million-Word Gap: Children's Conversational 
Exposure Is Associated With Language-Related Brain Function. Psychological Science, 29(5), 700-710. doi:10.1177/0956797617742725

[8] Al-Mahrooqi, R., Denman, C. J., \& Sultana, T. (2016). Factors Contributing to the Survival of Standard Arabic in the Arab World: An Exploratory Study (Vol. 24).

[9] Serbin, L. A., \& Karp, J. (2004). The Intergenerational Transfer of Psychosocial Risk: Mediators of Vulnerability and Resilience. Annual Review of Psychology, 55(1), 333-363. doi:10.1146/annurev.psych.54.101601.145228

[10] Opperman, A. (2016). The application of culturally-relevant instructional scaffolding techniques into english language learning (Order No. 10621816). Available from ProQuest Dissertations \& Theses Global. (1952230260). Retrieved from https://search.proquest.com/docview/1952230260?accountid=9758

[11] Bradley, R. H., \& Caldwell, B. M. (1984). The HOME Inventory and family demographics. Developmental Psychology, 20(2), 315.

[12] Achenbach, T. M., \& Rescorla, L. A. (2000). Manual for the ASEBA preschool forms and profiles (Vol. 30). Burlington, VT: University of Vermont, Research center for children, youth, \& families. Chicago.

[13] Diamond, A., \& Lee, K. (2011). Interventions Shown to Aid Executive Function Development in Children 4 to 12 Years Old. Science, 333(6045), 959-964.

doi:10.1126/science.1204529

[14] Binns, C. Field Report, 1001 Storytimes. Stanford Social Innovation Review Fall 2017. [15] Leadbeater, C. (2012). Innovation in Education. Bloomsbury. 